\title{
Induction of heme oxygenase- 1 attenuates lipopolysaccharide-induced inflammasome activation in human gingival epithelial cells
}

\author{
HONG LI, XIAOLI ZHOU and JIANLI ZHANG \\ Department of Stomatology, The First Affiliated Hospital, \\ Henan University of Science and Technology, Luoyang, Henan 471003, P.R. China
}

Received April 14, 2014; Accepted July 15, 2014

DOI: $10.3892 / \mathrm{ijmm} .2014 .1865$

\begin{abstract}
Interleukin-1 $\beta$ (IL-1 $\beta$ ) is a pathogenic factor for the destruction of periodontal tissue in periodontitis. The processing of IL- $1 \beta$ is regulated by cytosolic machinery termed as the inflammasome, which recruits and activates caspase- 1 and then cleaves pro-IL-1 $\beta$ to produce mature IL-1 $\beta$. Porphyromonas gingivalis ( $P$. gingivalis) infection and lipopolysaccharide (LPS) have been shown to activate the NLRP3 inflammasome and stimulate IL-1 $\beta$ production in human oral cells. Heme oxygenase-1 (HO-1) is an ubiquitous cytoprotective enzyme. The products of HO-1 exhibit protective biological activities, including antioxidant and anti-inflammatory effects. In the present study, we investigated the hypothesis that the induction of HO-1 inhibits the activation of the inflammasome and protects against LPS-induced inflammatory damage in cultured human gingival epithelial cells (GECs). Our results revealed that LPS induced the overexpression of the inflammasome components, NLRP3, apoptosis-associated speck-like protein containing a caspase recruitment domain (ASC) and caspase-1, by western blot analysis. Co-immunoprecipitation analysis indicated that LPS increased the binding of NLRP3 and ASC, and confocal imaging revealed that LPS increased the immunostaining and co-localization of ASC and caspase-1, indicating that LPS enhanced the assembly/formation of the inflammasome components. Hemin, a potent HO-1 inducer, blocked the LPS-induced overexpression and the formation of the NLRP3 inflammasome. Furthermore, hemin also inhibited the LPS-induced increase in the production of IL-1 $\beta$, as shown by enzyme-linked immunosorbent assay and blocked the nuclear translocation of pro-inflammatory nuclear factor- $\mathrm{\kappa B}$ (NF- $\mathrm{\kappa B})$, as shown by confocal assays. As a result, hemin protected the cells from LPS-induced damage, which was demonstrated by the immu-
\end{abstract}

Correspondence to: Dr Hong Li, Department of Stomatology, The First Affiliated Hospital, Henan University of Science and Technology, 24 Jinghua Road, Luoyang, Henan 471003, P.R. China E-mail: drlihongly@gmail.com

Key words: interleukin-1 $\beta$, hemin, E-cadherin, inflammation nostaining pattern of the cell junction protein, E-cadherin. LPS produced a disturbed staining pattern of E-cadherin, suggesting the disruption of epithelial integrity, which was abolished in the hemin-treated cells. In conclusion, our data demonstrate that the induction of HO-1 by hemin attenuates LPS-induced inflammatory damage in human GECs through the inhibition of inflammasome activation.

\section{Introduction}

Periodontitis, one of the most prevalent chronic diseases, is an infection-associated chronic inflammatory disease of the periodontium and the major cause of tooth loss. Although interleukin-1 $\beta$ (IL-1 $\beta$ ) is critical in the host defense against pathogens, it has been shown to be a pathogenic factor for the destruction of periodontal tissue in periodontitis $(1,2)$. The processing of IL- $1 \beta$ is regulated by cytosolic machinery termed as the inflammasome $(3,4)$. The major components of the inflammasome include the NOD-like receptor family, pyrin domain containing protein (NLRP) and apoptosis-associated speck-like protein containing a caspase recruitment domain (ASC), which recruits and activates caspase-1. Activated caspase-1 in turn cleaves pro-IL-1 $\beta$ to produce mature IL-1 $\beta$. Therefore, the inflammasome plays an important role in the production of IL-1 $\beta$.

Different inflammasomes, which contain different NLRP family proteins, have been identified, such as NLRP1, NLRP3, and NLRC4. These different inflammasomes are activated in response to different stimuli (5). It has been demonstrated that the NLRP3 inflammasome is the NLR subset inflammasome that is activated in periodontitis (6). It has also been shown that Porphyromonas gingivalis ( $P$. gingivalis) infection activates the NLRP3 inflammasome and increases the production of IL-1 $\beta$ (7-8). Similarly, the bacterial endotoxin, lipopolysaccharide (LPS), can also activate the NLRP3 inflammasome and stimulate the production of IL-1 $\beta$ (8-10). Gingival epithelial cells (GECs) are an important component of the innate host response to periodontal bacteria and make a significant contribution to the gingival health of the host (11). A recent study revealed that the LPS-induced activation of the NLRP3 inflammasome is an important mediator of the inflammatory response in the gingival epithelium (8). Therefore, targeting 
the inflammasome in the gingival epithelium may be a potential strategy for the prevention and treatment of periodontitis.

Heme oxygenase-1 (HO-1) is an ubiquitous inducible cellular stress protein and an endogenous cytoprotective enzyme (12). HO-1 catalyzes the rate-limiting step in heme degradation, producing equimolar quantities of biliverdin, iron and carbon monoxide. Biliverdin is subsequently converted to bilirubin by biliverdin reductase (13-15). The products of HO-1 exhibit protective biological activities, including antioxidant and anti-inflammatory effects (12). Reactive oxygen species have been shown to activate the inflammasome and antioxidants inhibit the inflammasome $(16,17)$. With a strong antioxidant function, the HO-1 pathway may inhibit the inflammasome. Indeed, although there are very limited reports studying the effect of the HO-1 pathway on the inflammasome, a literature search found two studies showing that the induction of HO-1 inhibited inflammasome activation and reduced the release of IL-1 $\beta$ in acute live failure (18) and lung injury (19). Given the fact that LPS produces oxidative stress and activates the inflammasome (8-10), we hypothesized that the induction of the HO-1 pathway may inhibit the inflammasome and protect against LPS-induced inflammatory damage in human GECs. To examine this hypothesis, we first determined the effects of hemin, a potent HO-1 inducer, on the LPS-induced activation of the inflammasome, then measured the production of IL-1 $\beta$ and the activation of nuclear factor $-\kappa \mathrm{B}(\mathrm{NF}-\kappa \mathrm{B})$, and finally, assessed the cell damage. To the best of our knowledge, our study is the first to demonstrate that the induction of HO-1 by hemin attenuates inflammatory damage in human GECs through the inhibition of inflammasome activation.

\section{Materials and methods}

Cell culture. Human GECs were purchased from Yuhengfeng Biotech (Beijing, China) and cultured according to the manufacturer's instructions in a defined epithelial cell medium (Yuhengfeng Biotech) supplemented with fetal bovine serum (FBS) (2\%), epithelial cell growth supplement (1\%), penicillin $(100 \mathrm{IU} / \mathrm{ml})$ and streptomycin $(100 \mu \mathrm{g} / \mathrm{ml})$ at $37^{\circ} \mathrm{C}$ in a humidified atmosphere of $5 \% \mathrm{CO}_{2}$ in air.

Cell treatment and experimental groups. The cells were subcultured in $10-\mathrm{cm}$ dishes and treated with the vehicle (0.2\% DMSO as a control), LPS $(2 \mu \mathrm{g} / \mathrm{ml})$, LPS plus hemin $(20 \mu \mathrm{mol} / \mathrm{l})$ or Ac-YVAD-CMK (10 $\mu \mathrm{mol} / 1$, a caspase- 1 inhibitor $)$ for $16 \mathrm{~h}$ when they reached $80 \%$ confluence. The cells were then harvested for protein and RNA isolation as described below. Some cells were cultured on glass coverslips for immunofluorescent staining and confocal microscopy as described below.

Preparation of cytosolic protein and nuclear extracts, western blot analyses for the protein levels of HO-1, NLRP3, ASC, caspase-1 and NF- $\kappa B$ (p65), as well as co-immunoprecipitation (Co-IP) of ASC and NLRP3. Cytosolic and nuclear proteins were prepared as previously described $(20,21)$. Briefly, the cells were scraped and washed with phosphatebuffered saline (PBS), and then homogenized in ice-cold HEPES buffer A containing $10 \mathrm{mM}$ HEPES (pH 7.9), $1.5 \mathrm{mM}$ $\mathrm{MgCl}_{2}, 10 \mathrm{mM} \mathrm{KCl}, 0.5 \mathrm{mM}$ dithiothreitol (DTT), $0.5 \mathrm{mM}$ phenylmethylsulfonyl fluoride (PMSF) and $10 \%$ Nonidet P-40.
Following centrifugation of the homogenate at $1,000 \mathrm{x} \mathrm{g}$ for $5 \mathrm{~min}$ at $4^{\circ} \mathrm{C}$, the supernatants were collected for cytosolic protein preparation and the pellets for nuclear protein isolation. The supernatants were centrifuged again at $6,000 \mathrm{x} g$ for $10 \mathrm{~min}$ and the resulting supernatants were used as cytosolic proteins for western blot analyses of HO-1, NLRP3, ASC and caspase-1.

For nuclear fraction isolation, the pellets from the first centrifugation, which contains cell nuclei, were washed with buffer $\mathrm{A}$ and then incubated with ice-cold HEPES buffer B containing $5 \mathrm{mM}$ HEPES (pH 7.9), $1.5 \mathrm{mM} \mathrm{MgCl}_{2}, 300 \mathrm{mM}$ $\mathrm{NaCl}, 400 \mathrm{mM} \mathrm{KCl}, 0.2 \mathrm{mM}$ EDTA, $0.5 \mathrm{mM}$ DTT, $0.5 \mathrm{mM}$ PMSF and $26 \%$ glycerol for $30 \mathrm{~min}$ to release nuclear proteins. Subsequently, the reaction mixtures were centrifuged at 23,000 rpm for $30 \mathrm{~min}$, and the supernatant was collected and stored at $-80^{\circ} \mathrm{C}$ until use as nuclear extracts for western blot analyses of NF- $\mathrm{BB}$ levels.

Western blot analysis was performed as previously described (22). Briefly, protein samples $(20 \mu \mathrm{g})$ were subjected to $10 \%$ sodium dodecyl sulfate-polyacrylamide gel electrophoresis (SDS-PAGE) and transferred onto nitrocellulose membranes. The membranes were probed with primary antibodies and then horseradish peroxidase-labeled secondary antibodies. Following incubation with enhanced chemiluminescence detection solution (ECL; Pierce, Rockford, IL, USA), the membranes were exposed to Kodak X-OMAT film (VWR International China Co., Ltd., Shanghai, China). The intensity of the blots on the film was determined using an imaging analysis program (ImageJ, free download from http:// rsbweb.nih.gov/ij/).

The primary antibodies used in the present study included anti-human HO-1 (1:1,000 dilution), NLRP3 (1:500), ASC $(1: 1,000)$, caspase-1 $(1: 3,000)$ and NF- $\mathrm{B}(\mathrm{p} 65)(1: 1,000)$, which were from Santa Cruz Biotechnology, Inc. (Dallas, TX, USA, distributor in Shanghai, China).

Co-IP of ASC and NLRP3. Co-IP was performed using a kit from Takara Bio, Inc. (Beijing, China) following the manufacturer's instructions. In brief, the cell lyses were mixed with antibody against ASC followed by the addition of protein-A beads. The beads were then collected and subjected to western blot analysis with anti-NLRP3 antibody.

Immunofluorescence microscopy. Immunofluorescence staining was performed using the cells cultured on glass coverslips, as previously described (20). Following fixation with $2 \%$ paraformaldehyde for $30 \mathrm{~min}$, the cells were incubated with anti-ASC (1:100 dilution), anti-caspase-1 (1:100 dilution), antiE-cadherin (1:200) and/or anti-NF- $\kappa \mathrm{B}(1: 200)$ antibodies at $4^{\circ} \mathrm{C}$ overnight. Following washing, the slides were incubated with corresponding Alexa Fluor 488- or Alexa Fluor 555-labeled secondary antibodies and then subjected to examinations using a confocal laser scanning microscope (FluoView FV1000; Olympus, Tokyo, Japan). The images were analyzed and the co-localization co-efficients were calculated using the computer program, Image-Pro ${ }^{\circledR}$ Plus (Media Cybernetics, Silver Spring, MD, USA), as previously described (23).

RNA extraction and reverse transcription-polymerase chain reaction (RT-PCR) of the $m R N A$ expression of $H O-1$. Total RNA 
A

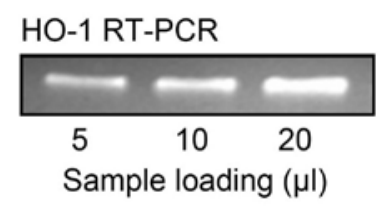

HO-1 Western blot analysis

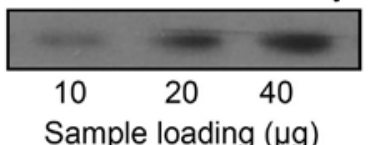

Sample loading $(\mu \mathrm{g})$

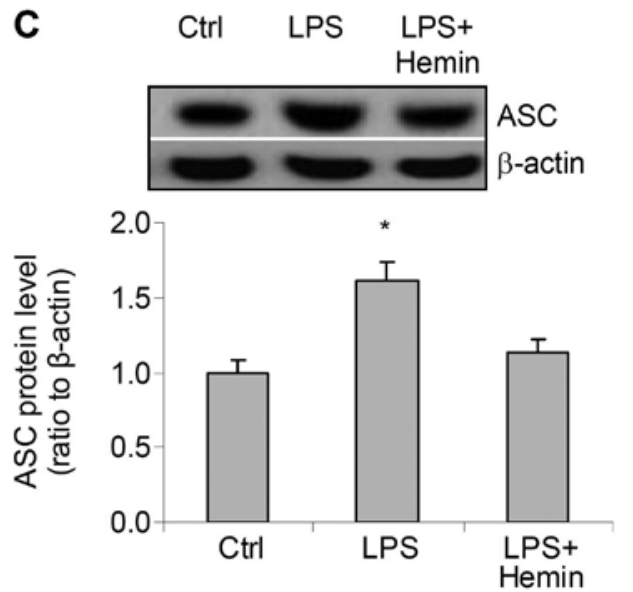

B
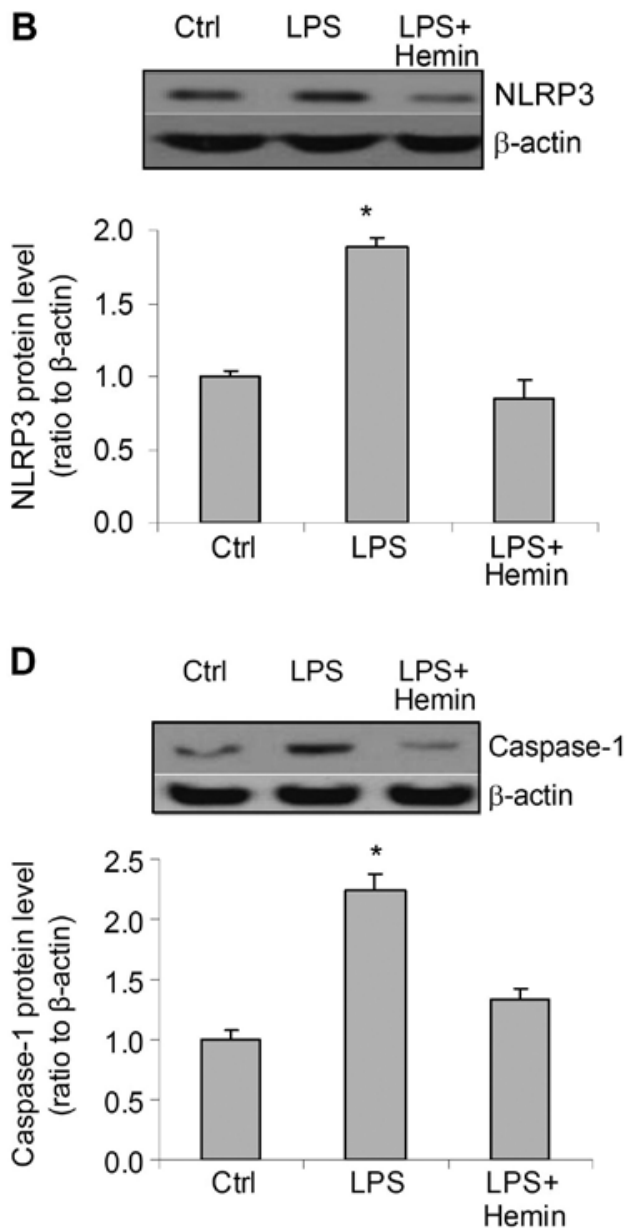

Figure 1. The expression of heme oxygenase-1 (HO-1) and the effects of hemin on the expression of the inflammasome components, NLRP3, apoptosis-associated speck-like protein containing a caspase recruitment domain (ASC) and caspase-1. (A) Representative gel documents showing HO-1 mRNA and protein expression by reverse transcription-polymerase chain reaction (RT-PCR) and western blot analyses. (B-D) Upper panels are representative western blot gel documents and lower panels are summarized blot intensities (normalized to control) showing the levels of inflammasome components. $\mathrm{n}=5$ batches of cells, ${ }^{*} \mathrm{P}<0.05 \mathrm{vs}$. other groups. Ctrl, control cells treated with the vehicle (0.2\% DMSO).

was extracted using TRIzol reagent (Life Technologies, Inc., Rockville, MD, USA). RNA was then reverse-transcribed and the PCR products were amplified using an RT-PCR kit (Solarbio Science and Technology, Beijing, China) and a thermocycler (Lab-Eye; Chuangmeng Biotechnology, Shanghai, China). The primers used were as follows: HO-1. sense, 5'-GGGTGACAGAAGAGGCTAAGACC-3' and antisense, 5'-AGATTCTCCCCTGCAGAGAGAAG-3' (24). The PCR cycling conditions were as follows: 30 cycles of $94^{\circ} \mathrm{C}$ for $30 \mathrm{sec} ; 55^{\circ} \mathrm{C}$ for $30 \mathrm{sec}$; and $72^{\circ} \mathrm{C}$ for $45 \mathrm{sec}$. The amplified products were visualized by $1.5 \%$ agarose gel electrophoresis, stained with ethidium bromide and images were captured under ultraviolet light.

Enzyme-linked immunosorbent assay (ELISA) of IL-1 $\beta$ in cell culture medium. The concentration of IL-1 $\beta$ in the cell culture medium was measured using a Valukine ELISA kit (R\&D Systems, Shanghai, China) according to the manufacturer's instructions.

Statistics. Data are presented as the means \pm standard error (SE). The significance of differences in mean values within and between multiple groups was evaluated by ANOVA followed by a Tukey's multiple range test. The Student's t-test was used to evaluate the statistical significance of differences between 2 groups. A value of $\mathrm{P}<0.05$ was considered to indicate a statistically significant difference.

\section{Results}

Effects of hemin on the expression of the inflammasome components, NLRP3, ASC and caspase-1. RT-PCR and western blot analysis of HO-1 mRNA and protein revealed the predicted bands, which confirmed that HO-1 was expressed in the cells used in the present study (Fig. 1A). Treatment of the cells with LPS markedly increased the protein levels of all 3 components of the inflammasome, NLRP3, ASC and caspase-1. However, hemin blocked the increase in the levels of NLRP3, ASC and caspase-1 induced by LPS (Fig. 1B-D). These data suggest that LPS stimulates the expression of inflammasome components and that hemin blocks the LPS-induced activation of the inflammasome.

Effect of hemin on the formation of the inflammasome. LPS markedly increased the immunostaining of caspase-1 and ASC (Fig. 2A), as well as the co-localization of these 


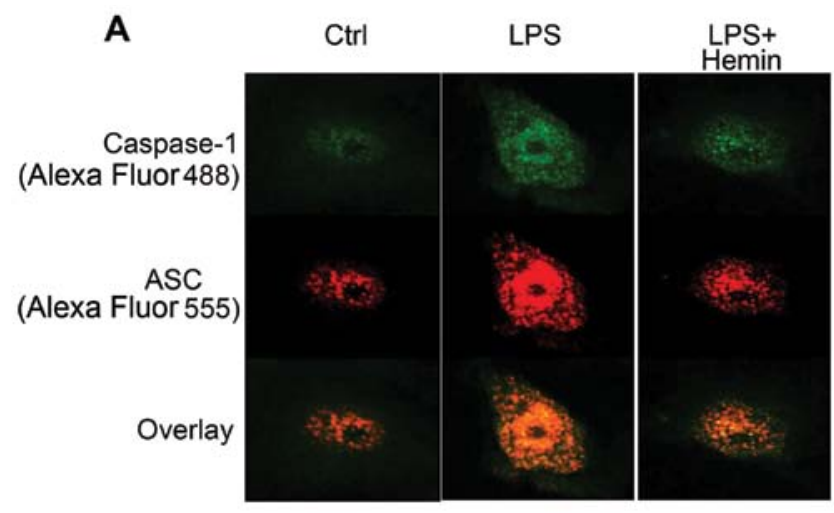

B

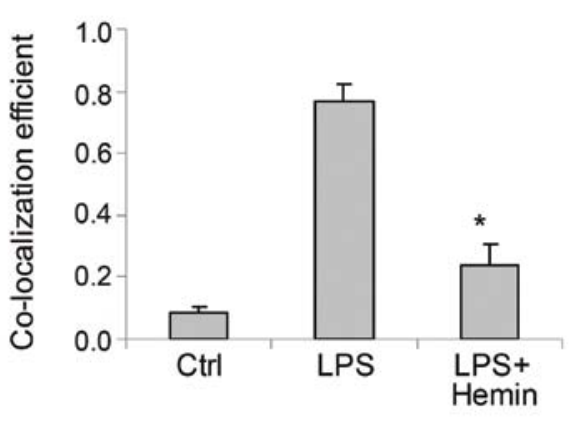

C

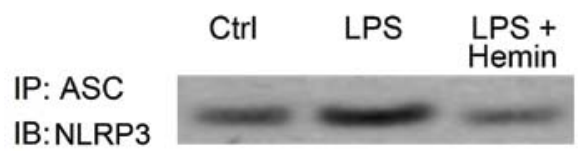

Figure 2. Effects of hemin on the formation of the inflammasome. (A) Representative confocal images showing immunostaining of caspase-1 and apoptosis-associated speck-like protein containing a caspase recruitment domain (ASC). (B) Co-localization co-efficients of caspase-1 and ASC overlays in panel A calculated using Image-Pro Plus software. (C) Representative gel document showing NLRP3 blots and co-immunoprecipitation (Co-IP) of ASC. $n=5$ batches of cells, ${ }^{*} \mathrm{P}<0.05$ vs. other groups. Ctrl, control cells treated with the vehicle $(0.2 \%$ DMSO)

2 proteins (Fig. 2B). (Co-IP) assay demonstrated that LPS markedly increased the binding of ASC with NLRP3 (Fig. 2C). The increased co-localization of caspase-1 and ASC, as well as the binding of NLRP3 and ASC were markedly inhibited by hemin (Fig. 2). These results indicated that LPS increased the assembly of inflammasome components and the recruitment of caspase-1, further suggesting that LPS activated the inflammasome and that hemin blocked the LPS-induced activation of the inflammasome.

Effects of hemin on the production of IL-1 $\beta$. In the LPS-treated cells, the levels of IL-1 $\beta$ were markedly increased; this effect was blocked by hemin (Fig. 3). These results suggested that hemin inhibited the LPS-induced activation of the inflammasome and reduced the production of IL-1 $\beta$. The inhibitory effects of hemin on the production of IL-1 $\beta$ were similar to those of YVAD, a caspase-1 inhibitor (Fig. 3), further indicating that treatment with hemin resulted in an inhibition of caspase-1 activity through the deactivation of the inflammasome.

Effects of hemin on the activity of the inflammatory factor, $N F-\kappa B$. Western blot analysis revealed a significant increase in the levels of NF- $\kappa \mathrm{B}$ in the nuclear extracts (Fig. 4A). Confocal

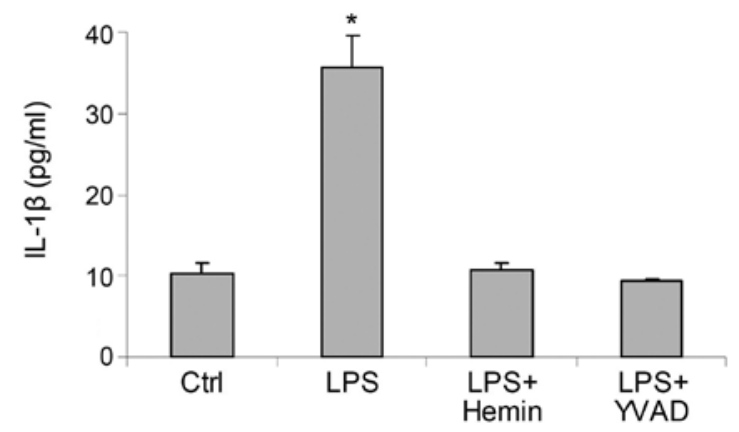

Figure 3. Effects of hemin on the interleukin-1 $\beta$ (IL-1 $\beta$ ) concentration in cell culture medium by enzyme-linked immunosorbent assay (ELISA). YVAD, Ac-YVAD-CMK, a caspase- 1 inhibitor. $n=4-6$ batches of cells, ${ }^{*} \mathrm{P}<0.05$ vs. other groups. Ctrl, control cells treated with the vehicle (0.2\% DMSO).

imaging indicated that NF- $\mathrm{B}$ was mainly located in the cytoplasm of the control cells (Fig. 4B), whereas in the LPS-treated cells, NF- $\kappa \mathrm{B}$ was mainly observed in the nucleus, indicating the activation and enhanced nuclear translocation of $\mathrm{NF}-\kappa \mathrm{B}$. These increases in the translocation of $\mathrm{NF}-\kappa \mathrm{B}$ into the nuclei were also blocked in the hemin-treated cells (Fig. 4).

Effects of hemin on cell damage. Confocal imaging revealed a derangement of the immunostaining pattern of the cell junction protein, E-cadherin, in the LPS-treated cells, which was blocked in the hemin-treated cells and in the cells treated with the caspase-1 inhibitor, YVAD, (Fig. 5), suggesting that the inhibition of the activation of the inflammasome and its downstream pro-inflammatory effectors by hemin protected the cells from LPS-induced damage.

\section{Discussion}

The present study demonstrated that the induction of HO-1 by hemin suppressed the LPS-induced increase in the expression of inflammasome components (NLRP3, ASC and caspase-1), inhibited the assembly of the NLRP3 inflammasome, and blocked the production of IL-1 $\beta$, as well as the activation of the pro-inflammatory factor, $\mathrm{NF}-\kappa \mathrm{B}$. As a result, hemin attenuated the LPS-induced cell damage in the cultured human GECs. To the best of our knowledge, the results of the present study are the first to suggest that the induction of HO-1 inhibits the activation of the inflammasome and protects human GECs against LPS-induced inflammatory damage.

Epithelial cells are the first line cells in contact with pathogens and danger signals/factors, including bacterial toxins. These cells also act as a physical barrier, protecting other cells, such as fibroblasts and osteoblasts, from exposure to pathogens. We thus selected to use GECs in the present study. We first confirmed the presence of HO-1 in the cells by detecting the mRNA and protein expression of $\mathrm{HO}-1$, which is consistent with previous studies showing that HO-1 is expressed in oral epithelial cells $(25,26)$. Similar to the results of a recent study (8), our results revealed an increased expression of NLRP3 inflammasome components in the GECs following treatment with LPS. Using confocal and Co-IP analyses, we further demonstrated that LPS not only increased the expression of NLRP3 inflammasome components, but also 
A
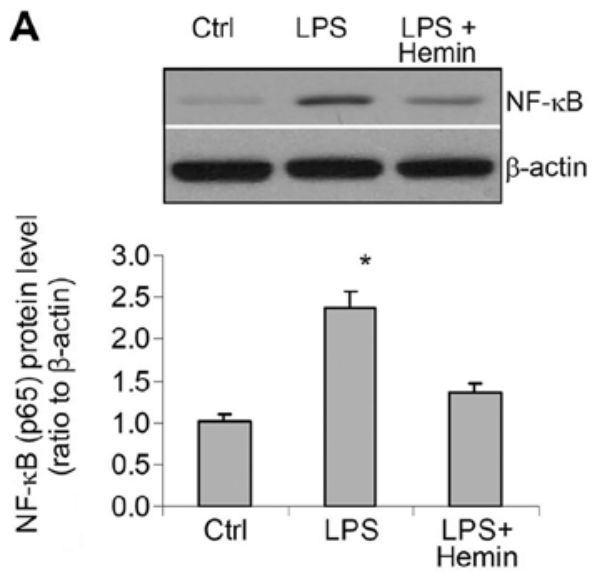

B
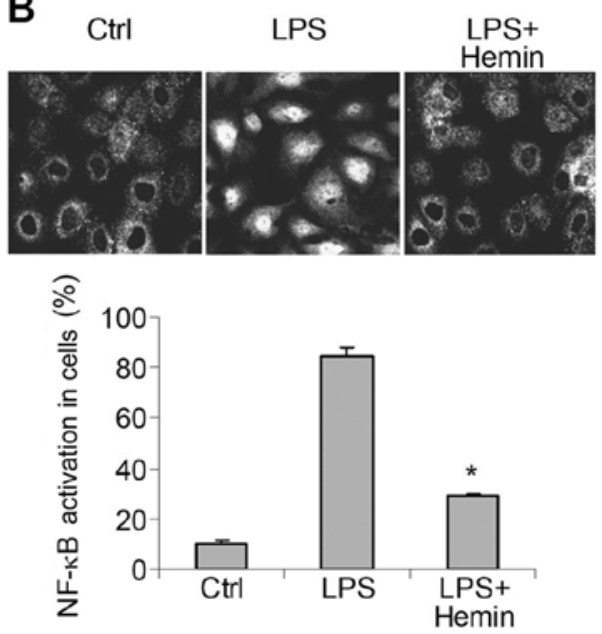

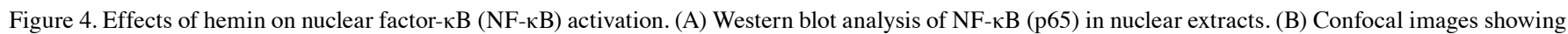
the localization of NF- $\mathrm{\kappa B}$ in the cells. $\mathrm{n}=4-5$ batches of cells, ${ }^{*} \mathrm{P}<0.05$ vs. other groups. Ctrl, control cells treated with the vehicle ( $0.2 \%$ DMSO).
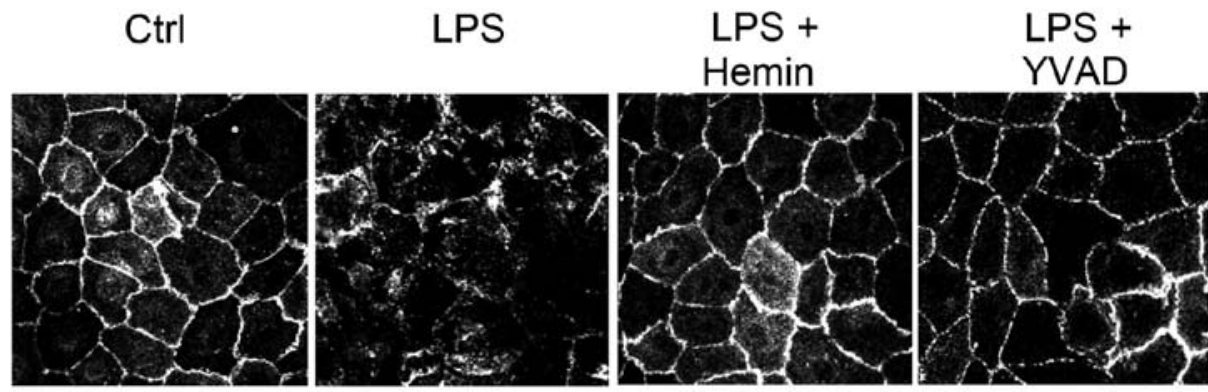

Figure 5. Effects of hemin on the immunostaining pattern of E-cadherin. Confocal images showing E-cadherin staining patterns. YVAD, Ac-YVAD-CMK, a caspase-1 inhibitor. Representative images from 5 batches of cells. Ctrl, control cells treated with the vehicle (0.2\% DMSO).

stimulated the assembly/formation of this machinery, further demonstrtating the activation of the NLRP3 inflammasome by LPS. Consequently, the production of IL-1 $\beta$ was increased in the LPS-treated cells. However, the LPS-induced activation of the NLRP3 inflammasome and the production of IL-1 $\beta$ were abolished by hemin, suggesting that the acvitation of HO-1 inhibits NLRP3 inflammasome activation, as well as IL-1 $\beta$ production in GECs.

Although it is well recognized that the induction of HO-1 exerts anti-inflammatory effects, the mechanisms involved have not yet been fully elucidated. The inhibition of inflammasome activation and IL-1 $\beta$ production may present one of the mechanisms through which HO-1 executes anti-inflammatory functions. In the present study, we did not attempt to explore the mechanisms through which the HO-1 pathway inhibits the LPS-induced activation of the NLRP3 inflammasome. In this regard, the antioxidant activities of HO-1 may be accountable for this effect. Oxidative stress has been shown to activate the inflammassome $(16,17)$, and at the same time, the products of HO-1 exhibit antioxidant functions (27). Thus, the mechanisms through which the HO-1 pathway inhibits the inflammasome may possibly be mediated through the antioxidant activities, as LPS has been shown to activate the inflammasome through oxidative stress $(9,10)$.
Since IL-1 $\beta$ has been shown to activate NF- $\mathrm{B}(28,29)$ and IL-1 $\beta$ mediates the LPS-induced activation of NF- $\kappa \mathrm{B}(10)$, we also evaluated the effects of hemin on the activation of $\mathrm{NF}-\kappa \mathrm{B}$ (p65). Our results demonstrated that LPS stimulated the translocation of $\mathrm{NF}-\kappa \mathrm{B}$ into the nucleus, as shown by the increased staining of $\mathrm{NF}-\kappa \mathrm{B}$ in the nucleus in the confocal images and the elevated levels of $\mathrm{NF}-\kappa \mathrm{B}$ in the nuclear extracts by western blot analysis. Hemin blocked the LPS-induced translocation of $\mathrm{NF}-\kappa \mathrm{B}$ into the nucleus. These data indicate that the activation of the HO-1 pathway blocks the activation of downstream inflammatory factors associated with the inflammasome. Since NF- $\kappa \mathrm{B}$, as a transcription factor, also stimulates IL-1 $\beta$ production (4), we cannot rule out the possibility that hemin directly inhibited the LPS-induced NF- $\kappa$ B activation in addition to its effect on the inflammasome and IL-1 $\beta$. Nevertheless, our data provide compelling evidence that the activation of the HO-1 pathway inhibits inflammasome activation and thereby blocks the downstream inflammatory response induced by LPS.

The inhibition of LPS-induced pro-inflammatory factors IL-1 $\beta$ and NF- $\kappa \mathrm{B}$ by hemin is expected to protect the cell damage. We then observed the immunostaining patter of a cell junction protein E-cadherin, which determines the epithelial integrity. In consistent with previous study (30), our result 
demonstrated that LPS disrupted cell junction as shown by the derangement of E-cadherin staining pattern. However, hemin blocked LPS-induced disturbance in E-cadherin staining pattern, suggesting that induction of HO-1 protected against LPS-induced inflammatory damage in gingival cells, probably through inhibition of LPS-induced activation of inflammasome.

In conclusion, the present study demonstrates that induction of HO-1 activity by hemin inhibits the LPS-induced activation of the NLRP3 inflammasome, reduces the resulting production of pro-inflammatory factors, and consequently attenuates cell damage. Thus, it can be concluded that the activation of HO-1 protects LPS-induced inflammatory damage in GECs, which may be used as a strategy for the prevention and treatment of chronic periodontitis.

\section{References}

1. Bascones A, Noronha S, Gómez M, Mota P, Gonzalez Moles MA and Villarroel Dorrego M: Tissue destruction in periodontitis: bacteria or cytokines fault? Quintessence Int 36: 299-306, 2005.

2. Orozco A, Gemmell E, Bickel $M$ and Seymour GJ: Interleukin-1beta, interleukin-12 and interleukin-18 levels in gingival fluid and serum of patients with gingivitis and periodontitis. Oral Microbiol Immunol 21: 256-260, 2006.

3. Pedra JH, Cassel SL and Sutterwala FS: Sensing pathogens and danger signals by the inflammasome. Curr Opin Immunol 21: $10-16,2009$.

4. Schroder K and Tschopp J: The inflammasomes. Cell 140: 821-832, 2010.

5. Broz P and Monack DM: Molecular mechanisms of inflammasome activation during microbial infections. Immunol Rev 243: 174-190, 2011.

6. Bostanci N, Emingil G, Saygan B, et al: Expression and regulation of the NALP3 inflammasome complex in periodontal diseases. Clin Exp Immunol 157: 415-422, 2009.

7. Park E, NaHS, Song YR, Shin SY, Kim YM and Chung J: Activation of NLRP3 and AIM2 inflammasomes by Porphyromonas gingivalis infection. Infect Immun 82: 112-123, 2014.

8. Yilmaz O, Sater AA, Yao L, Koutouzis T, Pettengill M and Ojcius DM: ATP-dependent activation of an inflammasome in primary gingival epithelial cells infected by Porphyromonas gingivalis. Cell Microbiol 12: 188-198, 2010.

9. Hua KF, Chou JC, Lam Y, et al: Polyenylpyrrole derivatives inhibit NLRP3 inflammasome activation and inflammatory mediator expression by reducing reactive oxygen species production and mitogen-activated protein kinase activation. PLoS One 8: e76754, 2013.

10. Kamo N, Ke B, Ghaffari AA, et al: ASC/caspase-1/IL-1ß signaling triggers inflammatory responses by promoting HMGB1 induction in liver ischemia/reperfusion injury. Hepatology 58: 351-362, 2013

11. Yilmaz O: The chronicles of Porphyromonas gingivalis: the microbium, the human oral epithelium and their interplay. Microbiology 154: 2897-2903, 2008.

12. Pae HO, Kim EC and Chung HT: Integrative survival response evoked by heme oxygenase- 1 and heme metabolites. J Clin Biochem Nutr 42: 197-203, 2008.

13. Maines MD: The heme oxygenase system: a regulator of second messenger gases. Annu Rev Pharmacol Toxicol 37: 517-554, 1997.
14. Ndisang JF, Tabien HE and Wang R: Carbon monoxide and hypertension. J Hypertens 22: 1057-1074, 2004.

15. Abraham NG and Kappas A: Heme oxygenase and the cardiovascular-renal system. Free Radic Biol Med 39: 1-25, 2005.

16. Villegas LR, Kluck D, Field C, et al: Superoxide dismutase mimetic, MnTE-2-PyP, attenuates chronic hypoxia-induced pulmonary hypertension, pulmonary vascular remodeling, and activation of the NALP3 inflammasome. Antioxid Redox Signal 18: $1753-1764,2013$

17. Komada T, Usui F, Shirasuna K, et al: ASC in renal collecting duct epithelial cells contributes to inflammation and injury after unilateral ureteral obstruction. Am J Pathol 184: 1287-1298, 2014.

18. Kim SJ and Lee SM: NLRP3 inflammasome activation in D-galactosamine and lipopolysaccharide-induced acute liver failure: role of heme oxygenase-1. Free Radic Biol Med 65: 997-1004, 2013.

19. Luo YP, Jiang L, Kang K, et al: Hemin inhibits NLRP3 inflammasome activation in sepsis-induced acute lung injury, involving heme oxygenase-1. Int Immunopharmacol 20: 24-32, 2014.

20. Han WQ,Zhu Q, Hu J, Li PL,Zhang F and Li N: Hypoxia-inducible factor prolyl-hydroxylase-2 mediates transforming growth factor beta 1-induced epithelial-mesenchymal transition in renal tubular cells. Biochim Biophys Acta 1833: 1454-1462, 2013.

21. Yu X, Deng L, Wang D, et al: Mechanism of TNF- $\alpha$ autocrine effects in hypoxic cardiomyocytes: initiated by hypoxia inducible factor $1 \alpha$, presented by exosomes. J Mol Cell Cardiol 53: 848-857, 2012.

22. Zhu Q, Wang Z, Xia M, Li P-L, Zhang F and Li N: Overexpression of HIF-1 $\alpha$ transgene in the renal medulla attenuated salt sensitive hypertension in Dahl S rats. Biochim Biophys Acta 1822: 936-941, 2012.

23. Zinchuk V, Zinchuk O and Okada T: Quantitative colocalization analysis of multicolor confocal immunofluorescence microscopy images: pushing pixels to explore biological phenomena. Acta Histochem Cytochem 40: 101-111, 2007.

24. Zhong Y, Liu T, Lai W, Tan Y, Tian D and Guo Z: Heme oxygenase-1-mediated reactive oxygen species reduction is involved in the inhibitory effect of curcumin on lipopolysaccharide-induced monocyte chemoattractant protein-1 production in RAW264.7 macrophages. Mol Med Rep 7: 242-246, 2013.

25. Tsai CH, Yang SF, Lee SS and Chang YC: Augmented heme oxygenase-1 expression in areca quid chewing-associated oral submucous fibrosis. Oral Dis 15: 281-286, 2009.

26. Milward MR, Chapple IL, Wright HJ, Millard JL, Matthews JB and Cooper PR: Differential activation of NF-kappaB and gene expression in oral epithelial cells by periodontal pathogens. Clin Exp Immunol 148: 307-324, 2007.

27. Haines DD, Lekli I, Teissier P, Bak I and Tosaki A: Role of haeme oxygenase-1 in resolution of oxidative stress-related pathologies: focus on cardiovascular, lung, neurological and kidney disorders. Acta Physiol (Oxf) 204: 487-501, 2012.

28. Tseng HC, Lee IT, Lin CC, et al: IL-1 $\beta$ promotes corneal epithelial cell migration by increasing MMP-9 expression through NF- $\mathrm{KB}$ and AP-1-dependent pathways. PLoS One 8: e57955, 2013.

29. Viñuales C1, Gascón S, Barranquero C, Osada J and Rodríguez-Yoldi MJ: Interleukin-1beta reduces galactose transport in intestinal epithelial cells in a NF- $\mathrm{KB}$ and protein kinase C-dependent manner. Vet Immunol Immunopathol 155: 171-181, 2013.

30. He D, Su Y, Usatyuk PV, et al: Lysophosphatidic acid enhances pulmonary epithelial barrier integrity and protects endotoxininduced epithelial barrier disruption and lung injury. J Biol Chem 284: 24123-24132, 2009. 